\title{
Crowd Management in der Lehre
}

\section{Rebekka Axthelm, Stefan Luppold und Marcus Moroff}

Wer schon einmal dicht gedrängt vor der Konzertbühne stand kann sich die aussichtslose Lage, wenn die Stimmung kippt und Panik aufkommt, gut vorstellen. Es ist sehr wichtig, Räume und Events, die zeitweise von sehr vielen Menschen aufgesucht werden, so zu gestalten und zu planen, dass maximale Sicherheit gewährleistet ist. Damit eine öffentliche Veranstaltung reibungslos verläuft ist eine gründliche Planung, also ein qualitativ hochwertiges Crowd Management unabdingbar.

\subsection{Ausgangssituation}

An der Dualen Hochschule Baden-Württemberg (DHBW) werden in branchenspezifischen Modulen Studiengangs „BWL - Messe-, Kongress- und Eventmanagement" unter anderem Konzepte des Crowd Management unterrichtet. Dabei ist es nicht nur wichtig Vorschriften zu kennen und anwenden zu können, sondern auch

R. Axthelm (凶)

Fakultät Informatik, (HTWG) Hochschule für Technik, Wirtschaft und Gestaltung

Konstanz, Konstanz, Deutschland

E-Mail: rebekka.axthelm@htwg-konstanz.de

S. Luppold

BWL- Messe- und Eventmanagement, Duale Hochschule Baden-Württemberg, Ravensburg, Deutschland

E-Mail: luppold@dhbw-ravensburg.de

M. Moroff

PerEx GmbH Stuttgart, Stuttgart, Deutschland

E-Mail: marcus.moroff@ perex.de 
bereits theoretisch eine realistische Vorstellung für verschiedene Situationen zu entwickeln. Die Studierenden wollen wir mit verschiedenen Methoden wirksam stützen und ausbilden, ohne sie bewusst einer Gefahr auszusetzen. Jeder Theorie muss im Studium zwingend die Darstellung, besser noch das Erleben der Praxisanwendung folgen. Dann erkennen die angehenden Veranstaltungsleitenden den Wert der theoretischen Grundlagen für das Berufsleben.

Der Anspruch des Seamless Learning, einen durchgehenden Roten Faden zu liefern, kann ein Garant dafür sein, dass sich Themen nachhaltig festsetzen und weiterentwickeln. Vor diesem Hintergrund bot sich das hochaktuelle Thema Crowd Management zur Entwicklung eines Seamless Learning-Stranges an.

Crowd Management ist die systematische Planung und proaktive Umsetzung der räumlichen Organisation von großen Menschenansammlungen auf Basis kontinuierlicher Überwachung und Analyse der Massenbewegungen und Gruppendynamiken mit dem Ziel der Sicherung, des Schutzes und des Erhalts des Wohlbefindens aller Anwesenden und Beteiligten. (Runkel \& Pohl, 2012)

Hierauf Bezug nehmend wollen wir einem Bruch "fehlender Übergang von theoretischen Kenntnissen und praktischen Erfahrungen" im Themenbereich Eventsicherheit (hier Synonym für die Sicherheit bei Messen, Ausstellungen, Kongressen, Tagungen, Marketing-Events etc.) begegnen. Für die Umsetzung wurden bestimmte Lehrinhalte und -konzepte geschaffen, die teilweise durch speziell entwickelte digitale Medien unterstützt werden. Die DHBW als duale Hochschule steht für besondere Praxisintegration während des Studiums und ist bestrebt, das Lehrangebot im Bereich E-Learning permanent weiterzuentwickeln. Das Arbeiten mit einer Simulationssoftware im Bereich Personenströme ist zuvor noch nicht durchgeführt worden. Eventplanende arbeiten im Beruf auch mit Simulationsprogrammen, haben diese aber im Studium nicht kennenlernen können. In Zusammenarbeit mit der Hochschule für Technik Wirtschaft und Gestaltung Konstanz (HTWG) wurde von der DHBW im Rahmen des Projektes das Lernobjekt $\mathrm{Cman}_{\text {event }}$ entwickelt, das eine teilweise softwarebasierte interaktive Lernumgebung mit begleitenden Unterrichtsmaterialien bereitstellt. Lernende können sich in einer äußerst praxisnahen Umgebung mit stets aktuellem Anwendungsbezug mit ihren Themen auseinandersetzen. Dies schafft eine Brücke in ihrer Lernbiographie und unterstützt die konsequente Verzahnung des wissenschaftlichen Studiums mit anwendungsbezogenem Lernen in der Arbeitswelt.

Die hier beschriebene Teilaufgabe des Projektes befasst sich mit der Erarbeitung einer didaktischen Lehrunterlage und ihrer Erprobung im Studienbetrieb des Studiengangs „BWL - Messe-, Kongress- und Eventmanagement“ der DHBW. 
Die Lehreinheit wurde im vierten Semester durchgeführt, umfasste zwei von sechzehn Lektionen und wurde von ungefähr dreißig Studierenden besucht.

Der didaktische Ansatz ergibt sich aus der Handhabung des Fachthemas Crowd Management in der Tagespraxis. Erst bei größeren Menschengruppen wird aktuell wirklich über Crowd Management nachgedacht, obwohl bei jedem Zusammentreffen von Menschen Elemente einer Besucherlenkung und einer Achtsamkeit für deren Unversehrtheit zum Zuge kommen sollte. Unterlagen in 2D prägen von der ersten Skizze bis zum maßstäblichen Plan den Entstehungsprozess von Veranstaltungen und Events in fast allen Ausprägungen. Das ist gelernt und wird angewandt. Mit Besichtigungen vor Ort in der Veranstaltungsstätte verschaffen sich die Planer bei sogenannten Site Inspections einen visuellen, emotionalen Eindruck, der in die Planungen einfließt und diese gegebenenfalls auch grundlegend verändert. Das betrifft sowohl den fachlich-inhaltlichen Teil der Eventplanung wie auch die sicherheitsbezogenen Abwägungen. Oft wirken die Eventplanenden überregional und ein visueller Zugriff auf die vorgesehene Location ist nur mit Aufwand möglich. Daraus erwuchs der Wunsch die 2D-Welt zu verlassen und sich mittels 3D weitere Gestaltungsmöglichkeiten zu erschließen. Seinen Ursprung hatte dieser Ansatz in der Eventsicherheitsplanung, die frühzeitig klären sollte, ob Wegeführungen, platzierte Gestaltungselemente, Sichtachsen, etc. ein krisensicheres Bewegen der anvertrauten Menschen möglich machen. Im weiteren Verlauf identifizieren die Kreativplanenden die sich daraus ergebenden Möglichkeiten und erweiterten den Anforderungskatalog an das Crowd Management um Elemente zum Darstellen der umzusetzenden Eventelemente für das Klientel (erlebbare Entscheidungsfindung bei der Kundschaft). Im vorliegenden Teilprojekt wurde diese Anforderungswelt den Studierenden schrittweise nähergebracht.

Plastische Modellhäuser und Figuren auf den 2D-Plänen regen die Phantasie an und erlauben ein gedankliches Eintauchen in das zu erwartende Erleben des Events. Dabei werden jedoch schnell die Grenzen dieses Verfahrens sichtbar. Änderungen der Parameter erfordern aufwendige Umbauten. Spezifische Rahmenbedingungen wie unvorhersehbare Ereignisse (Wetter, Lichteinfall, etc.) sind nicht oder nur höchst unzureichend abbildbar. Der Ruf nach einer „,maschinellen Lösung“ erfolgt fast reflexartig. Gefragt waren nun mathematische Ansätze, die das Abbilden der Lebenswirklichkeit in Modellen ermöglichen. Die Simulationssoftware pFlow mit webbasierter Bedienoberfläche wurde an der ZHAW und an der HTWG entwickelt (s. Axthelm, 2016, S. 233). Erweitert werden 3D-Lösungen zunehmend um Elemente der Virtual Reality (VR), die ein erlebbares Eintauchen des Planenden in die geschaffene Eventwelt ermöglichen. Mit den Studierenden wurden die aus diesem Teilprojekt hervorgegangenen Simulationssettings erprobt. 


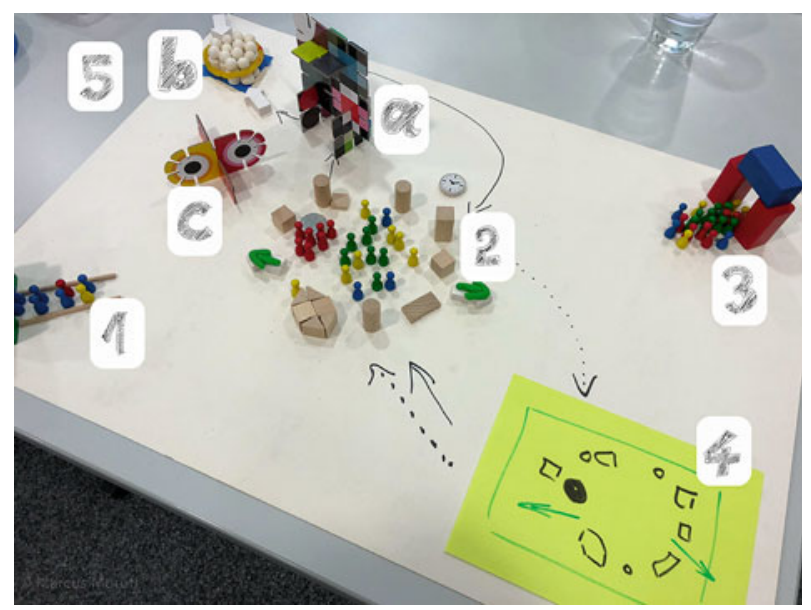

Abb.5.1 Visualisierung des strukturellen Aufbaus des Lernobjekts

Dass das Crowd Management ein Planungskonzept für die Sicherheit von Menschen ist muss heute immer noch bei vielen Veranstaltern betont werden. Das Selbstverständnis hierfür ist nicht von vornherein gegeben und damit ist auch die Kommunikation von Planenden mit Veranstaltenden ein wesentlicher Bestandteil des Eventmanagements (s. Luppold \& Moroff, 2019).

\subsection{Konzept}

In Zusammenarbeit mit der Universität St. Gallen (USG) wurde im Rahmen mehrerer Workshops der Roten Faden des Unterrichtsgerüsts erarbeitet. Abb. 5.1 zeigt den zweidimensionalen Plan, wie er heute Grundlage der Eventgestaltung ist. Er beinhaltet neben den Grundlagen des Crowd Management auch ein reales Erleben am eigenen Körper und Simulationen bestimmter Szenarien am Computer, welche sich auch zu einem VR-gestützten Erleben ausbauen lassen. Durch die computergestützten und realitätsnahen Simulationen lässt sich die Notwendigkeit des Crowd Management anhand konkreter Vorkommnisse aufzeigen. Die angesetzten Lernziele der Unterrichtseinheit sind folgendermaßen gewählt:

- Die Lernenden können das Phänomen „Menschen im Raum“ mit eigenen Worten beschreiben. 
- Die Lernenden können Einflussfaktoren auf Menschen im Raum, je nach Situation, aufzählen und erläutern.

- Die Lernenden können Steuerungsmöglichkeiten benennen und deren Wirkung aus eigener Erfahrung beschreiben.

- Die Lernenden können manuelle Planungsinstrumente aufzählen.

- Die Lernenden können alle Schritte von der Idee der Planung bis zur Anwendung gliedern und erörtern.

Die aufgezählten Lernziele sind nach Still (2013) in zwei Strukturebenen eingeflochten: Das ist zum einen die Phaseneinteilung in Ingrees (Ankunft), Circulation (Verlauf) und Egrees (Auslass) und zum anderen eine kategorische Einteilung in Design (geographische Gegebenheiten der Veranstaltung), Information (Tafel, Mikrofon etc.) und Management (Personal, Beschilderungen, Abläufe, etc.).

Im ersten Workshop mit der USG, dem Anforderungsworkshop wurden zwei Brüche dieser Lehreinheit herausmodelliert. Der Bruch „Vorstellung-Realität“ beschreibt die Schwierigkeit, auf einer rein theoretischen Ebene Situationen nachvollziehbar zu machen, die man nur am eigenen Leib in einer dichten Menschenmenge erfahren kann. Der Bruch „Theorie-Praxis“ zielt auf den Umstand ab, dass Eventplaner in der Praxis oft mit Simulationstools arbeiten, im Studium aber mit dieser Möglichkeit nicht in Berührung kommen.

Im zweiten, dem Prototypenworkshop wurde das Unterrichtsgerüst erarbeitet. Dieses dient als Grundlage des strukturellen Aufbaus der Lehreinheit. Abbildung 1 zeigt den Roten Faden dieses Gerüsts. Die Eckpunkte, die im Workshop erarbeitet wurden und als Ausgangslage des iterativen Prozesses der Unterrichtsgestaltung dienten, sind:

- Aufzeigen der Notwendigkeiten zum Crowd Management anhand realer Vorkommnisse

- Grundlagen des Crowd Management: Einflussfaktoren erkennen, definieren und umsetzen

- Der zweidimensionale Plan ist heute die Grundlage der Eventgestaltung

- 3D-Umwandlungen und VR-gestütztes Erleben der Planung im Raum sind im Kommen

- Mathematische Modelle ermöglichen das Aufzeigen von berechenbaren Strukturen (Softwarelösungen)

Das hier vorgeschlagene Unterrichtskonzept basiert auf dem Design Based Research (DBR) Ansatz (zitiert in Dilger et al., 2019, S. 366) und arbeitet mit zwei didaktischen Prinzipien: Der Zugang wird über ein problembasiertes Lernen 
aufgebaut, in welchem ganz konkrete Fragestellungen der Praxis im Vordergrund stehen. „Wie ist die reale Situation?“, „Welche Szenarien müssen beachtet werden?" etc. Die Durchführung stützt sich dann stark auf spielbasiertes Lernen. Dabei geht es nicht darum, Punkte zu sammeln und am Ende das Spiel zu gewinnen, sondern viel mehr, die Situation mit Spielfiguren zu modellieren und Möglichkeiten zu schaffen, dass Lernende auf verschiedene Weise ,in die gedachte, reale Situation“ hinein zu versetzen. Dazu werden dann als technologische Werkzeuge sowohl digitale als auch ganz konkrete, reale Hilfsmittel eingesetzt. Das Spielerische bezieht sich auch auf die körperliche Aktivierung der Lernenden aus der rein auditiven Lernposition heraus.

Abb. 5.1 zeigt ein Modell der Unterrichtseinheit. Es beinhaltet die Veranschaulichung der realen Situation mit der Struktur des Unterrichtablaufs. In verschiedenen Stufen und mit verschiedenen Medien wird den Studierenden ein Blick auf mögliche Szenarien und ein Vorstellungsvermögen der jeweiligen Situationen gegeben.

Die Diagonale von links unten [1] über die Mitte [2] nach rechts oben [3] zeichnet die reale Situation: [1] Personen betreten den betrachteten Raum in geordneter Struktur und begehen den eigentlichen Eventbereich. Die abgebildete Uhr deutet an, dass je nach Uhrzeit unterschiedliche Nutzungsverhalten vorliegen: Das Eintreffen der Personen zu Beginn [1], der Verlauf der Festivität [2] und das Verlassen des Geländes am Ende [3]. Letzteres kann einem Evakuierungsszenario entsprechen. Generell hängt jede Phase von der Art der Veranstaltung ab.

Die Diagonale von rechts unten [4] über die Mitte [2] nach links oben [5a-c] zeichnet die Ausbildungsschritte der Studierenden in dieser Unterrichtseinheit: [4] Die erste Planungsidee wird zum zweidimensionalen Plan als Skizze auf Papier. [2] Zur Verbesserung der räumlichen Vorstellung der Veranstaltung wird mit Figuren und Spielhäusern eine dreidimensionale Welt geschaffen. Darin gibt es attraktive Bereiche, in denen sich Ballungen entwickeln, was durch rote Figuren kennzeichnet ist, und es gibt Bereiche, in denen gar nichts passiert und dann diejenigen mit mäßigem Betrieb; hier durch gelbe und grüne Figuren sichtbar gemacht. Abb. 5.2 zeigt die Umsetzung dieses Teils im Unterricht. Die Eventplanung sieht nun vor, möglichst wenige dieser roten Bereiche auftreten zu lassen. Das Handwerkszeug einer guten Konzeptionierung erhalten Studierende an der Hochschule [5]. Die Wissensvermittlung findet dort auf drei Ebenen statt:

a) Theoretisches Wissen

b) Erfahrung am eigenen Leib

c) Berechnungsmöglichkeiten und Erfahrungen mithilfe digitaler Medien 


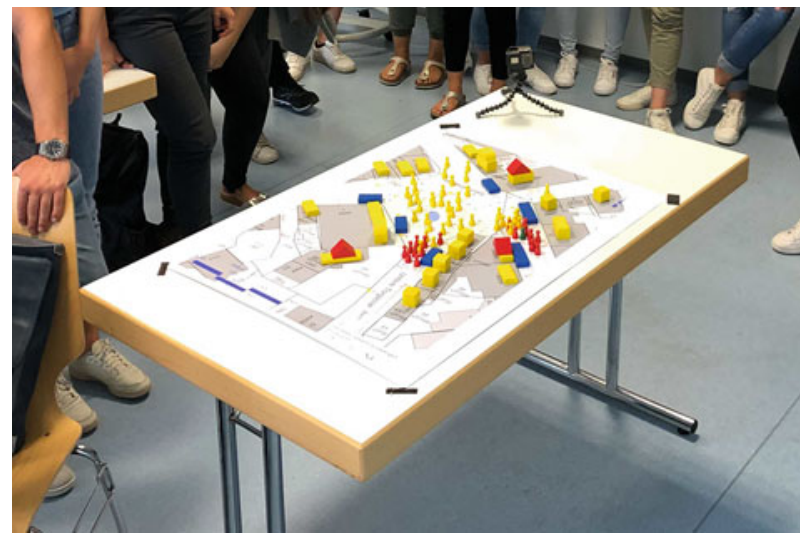

Abb.5.2 Reales 3D-Modell eines öffentlichen Platzes

Und genau hier, bei der Verbindung von Theorie-Realität-Digitalisierung verbergen sich die Seams des ,Seamless Learning“-Konzepts und das in zweierlei Hinsicht: Das Unterrichtskonzept verbindet zum einen Theorie und Realität und zum anderen werden Studierende an die Nutzung von digitalen Medien herangeführt wie sie sie später im Berufsleben weiterhin nutzen können. Präziser: Neben dem theoretischen Grundlagenwissen, worauf sich die Wissensvermittlung im Unterricht bisher im Wesentlichen stützte, sollten Planende ein Gefühl dafür bekommen, was es bedeutet, wenn zwei oder zehn Personen auf einen Quadratmeter dicht gedrängt sind. Es ist eine beeindruckende Erfahrung, wenn man sich in einer Gruppe aus zehn bis zwölf Personen befindet, die durch ein Seil [5b] zusammengehalten wird. Das Seil bleibt natürlich lose, sodass keine Gefahr besteht und damit ist die Situation nicht gleich eine solche, in der es kein spontanes Entkommen gibt. Dennoch bewirkt dieses Experiment eine verbesserte Vorstellung.

[5c] Ein weiterer Weg zur verbesserten Vorstellungskraft ist das Arbeiten mit digitalen Medien. Anhand mathematischer Modelle lässt sich die Umgebung realistisch konstruieren. Die Bewegung der Akteure und ihr voraussichtliches Verhalten ist berechenbar und parametrisiert steuerbar. Abb. 5.3a zeigt eine webbasierte $^{1}$ Simulation eines Evakuierungsszenarios mit zwei Ausgängen, die unterschiedlich bevorzugt werden, sowie attraktive und unattraktive Bereiche. Passend zu dieser Unterrichtseinheit wurden an der HTWG bestimmte Settings

\footnotetext{
${ }^{1}$ https://www.ios.htwg-konstanz.de/modelling-and-simulation-workgroup.
} 

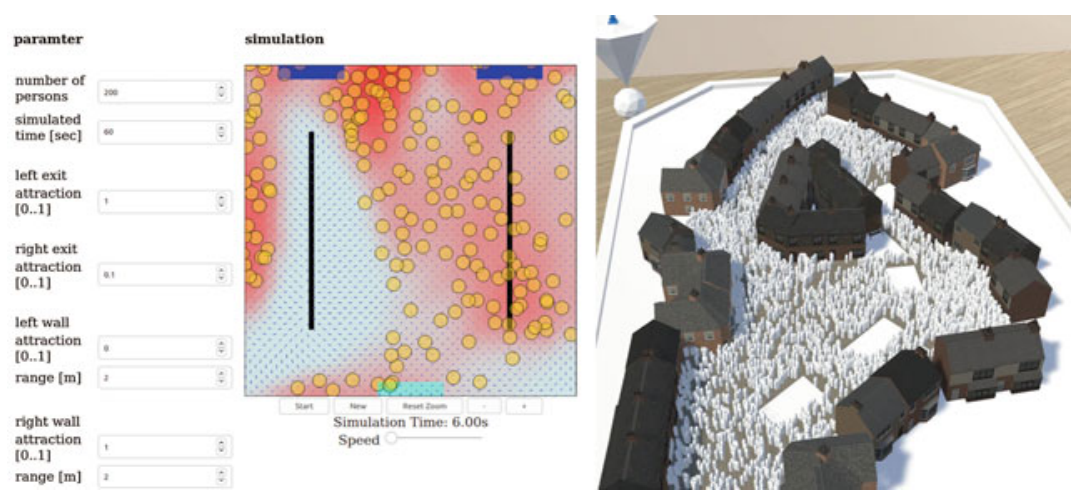

Abb.5.3 a Computersimulation von Personenströmen (links); b Visualisierung der Berechnungen in einer VR (rechts)

zur Simulation von Personenströmen aufgestellt. Studierende können verschiedene Parameter wählen, damit unterschiedlichste Szenarien aufsetzen und die entsprechenden Verläufe studieren. Es lassen sich in der Software zwar nicht selbstständig neue Settings (etwa ein Fußballstadion statt eines Restaurants) aufsetzen aber die vorhandenen Szenarien lassen sich ohne große Einarbeitung verwenden.

Abb. 5.3b zeigt einen öffentlichen Platz, für den der Zürcher Münsterplatz als Vorlage diente, der Simulationsergebnisse von Fußgängerströmen in einer VR visualisiert. Planende können in verschiedene Rollen ein tauchen und sich virtuell im Raum bewegen. Mit diesem Tool ist es möglich, nicht nur Abläufe vorherzusagen, sondern mitten im Raum zu stehen und somit den Eindruck von Varianten der Personendichte noch besser interpretieren zu können. Die VR-Software muss lokal installiert werden und benötigt bestimmte Hardware-Komponenten.

\subsection{Erkenntnisse und Fazit}

Zu Beginn der Forschungsarbeit und an deren Ende wurden in Evaluationsrunden mit den Studierenden deren Erfahrungen und Lernerfolge diskutiert. Die Studierenden der Einstiegsevaluation verfügten über Kenntnisse der Eventplanung und -durchführung. Sie verhielten sich zielorientiert und einzelthemenbezogen. Ein Anspruch auf eine thematische Durchgängigkeit war nicht erkennbar. 
Die eher subjektiven Erkenntnisse zeigen, dass es ganz entscheidend ist, das Interesse der Lernenden zu wecken. Das gelingt sehr gut wenn man die Parallelen zur Gaming-Welt aufzeigt und gleichzeitig den Spieltrieb der Lernenden weckt. Dies zieht sich als roter Faden nahtlos von der reinen Vorstellung über die Spielfiguren und eine Software, die einen mathematischen Ansatz verfolgt bis hin zur konkreten Planung, wie sie im späteren Berufsleben erstellt werden muss.

Kritisch ist, ein steter Begleiter aller Doziernden, die positive Korrelation von Unterrichtszeit und der Anzahl spielerischer Elemente in der Lehre.

Die Bedienung einer Software setzt natürlich voraus, dass man sich der Parameter, die man verwendet, ein wenig bewusst ist. Als lehrreich kann man es dahin gehend verstehen, dass das auch für lizenzierte Software gilt, die professionell eingesetzt werden. Ein weiteres Problem ergibt sich daraus, dass üblicherweise die Tools zur Personensimulation nicht frei verfügbar, sondern kostenpflichtig sind.

Die abschließende Evaluation zeigt, dass es gelungen ist, den Studierenden den Roten Faden durch das Thema didaktisch aufzuzeigen. Dieses erfreuliche Ergebnis gewinnt an Bedeutung, wenn man bedenkt, dass es unter pandemiebedingten Gegebenheiten der Online-Vorlesung zustande gekommen ist.

\section{Literatur}

Axthelm, R. (2016). Finite element simulation of a macroscopic model for pedestrian flow. In W. Daamen \& V. L. Knoop (Hrsg), Traffic and granular flow '15 (S. 233-240). Springer. https://doi.org/10.1007/978-3-319-33482-0

Dilger, B., Gommers, L. Rapp, Ch., Trippel, M., Butz, A., Huff, S., Mueller, R., \& Schimkat, R. (2019). Seamless Learning als Ansatz zum Umgang mit flexiblem Lehren und Lernen - Erfahrungsbericht aus dem Seamless Learning Lab. In C. Müller, P. Barthelmess, C. Berger, G. Kucza, M. Müller, \& P. Sieber (Hrsg.), Zeitschrift für Hochschulentwicklung (S. 361-376). Graz. https://doi.org/10.21256/zhaw-18756.

Luppold, S., \& Moroff, M. (2019). Crowdmanagement bei Events: Verbesserung der Sicherheitsmaßnahmen, Förderung eines ganzheitlichen Verständnisses und Kreation von wirkungszentrierten Szenarien durch Ex-Ante-Simulation. In C. Zanger (Hrsg.), Eventforschung. Markenkommunikation und Beziehungsmarketing (S. 289-295). Springer Gabler. https://doi.org/10.1007/978-3-658-27652-2_17.

Runkel, S., \& Pohl, J. (2012). Crowd management als Planungsaufgabe. Eine sozialgeographische Perspektive auf Masse und Raum bei Großveranstaltungen. In Geographische Zeitschrift (Bd. 100, S. 189-207). Franz-Steiner-Verlag. Heft 4.

Still, K. G. (2013). Introduction to crowd science. Taylor \& Francis Group. 
Open Access Dieses Kapitel wird unter der Creative Commons Namensnennung 4.0 International Lizenz (http://creativecommons.org/licenses/by/4.0/deed.de) veröffentlicht, welche die Nutzung, Vervielfältigung, Bearbeitung, Verbreitung und Wiedergabe in jeglichem Medium und Format erlaubt, sofern Sie den/die ursprünglichen Autor(en) und die Quelle ordnungsgemäß nennen, einen Link zur Creative Commons Lizenz beifügen und angeben, ob Änderungen vorgenommen wurden.

Die in diesem Kapitel enthaltenen Bilder und sonstiges Drittmaterial unterliegen ebenfalls der genannten Creative Commons Lizenz, sofern sich aus der Abbildungslegende nichts anderes ergibt. Sofern das betreffende Material nicht unter der genannten Creative Commons Lizenz steht und die betreffende Handlung nicht nach gesetzlichen Vorschriften erlaubt ist, ist für die oben aufgeführten Weiterverwendungen des Materials die Einwilligung des jeweiligen Rechteinhabers einzuholen.

(c) (1) 